\title{
The Child-Langmuir law in the quantum domain
}

\author{
Debabrata Biswas and Raghwendra Kumar
}

Theoretical Physics Division, Bhabha Atomic Research Centre, Mumbai 400 085, INDIA

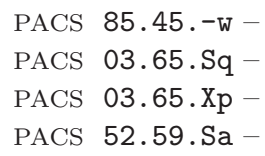

\begin{abstract}
It is shown using dimensional analysis that the maximum current density $J_{Q C L}$ transported on application of a voltage $V_{g}$ across a gap of size $D$ follows the relation $J_{Q C L} \sim$ $\hbar^{3-2 \alpha} V_{g}^{\alpha} / D^{5-2 \alpha}$. The classical Child-Langmuir result is recovered at $\alpha=3 / 2$ on demanding that the scaling law be independent of $\hbar$. For a nanogap in the deep quantum regime, additional inputs in the form of appropriate boundary conditions and the behaviour of the exchange-correlation potential show that $\alpha=5 / 14$. This is verified numerically for several nanogaps. It is also argued that in this regime, the limiting mechanism is quantum reflection from a downhill potential due to a sharp change in slope seen by the electron on emerging through the barrier.
\end{abstract}

Introduction. - The Child-Langmuir (CL) law [1] the cornerstone of classical space charge limited flows. It determines the maximum current $\left(J_{C L}\right)$ that can flow cross a gap of size $D$ on application of an electric field $-V_{g} / D$, given the existence of mono-energetic electrons at the cathode [2, 3]. The limiting mechanism is fairly straightforward: the mutual repulsion between the electrons gives rise to a barrier in the net electrostatic potential and an energy spread in the initially mono-energetic beam of electrons. Beyond the limiting current, the excess electrons have energy slightly lower than the barrier height and are thus reflected back to the cathode. This picture holds for electrons with zero energy as well (relative to the cathode potential) where a more common, albeit equivalent, criterion of the limiting mechanism is the total cancellation of the applied field by the space charge field at the cathode and can thus be arrived at from the Poisson equation of electrostatics.

The quantum treatment for studying steady state charge transport across a gap is based on a self-consistent solution of the Schrödinger and Poisson equations [4, 5]. As in the classical case, there exists a maximum current in this formalism beyond which a solution to the coupled set of equations ceases to exist. The exact limiting mechanism is however largely uninvestigated with the studies largely centred around scaling laws.

The purpose of this letter is to (i) provide a unified scaling law across all regimes whenever a power law behaviour holds, (ii) probe the deep quantum regime using additional inputs such as the boundary conditions and the behaviour of the exchange-correlation potential and (iii) investigate the limiting mechanism in the quantum domain.

The model that we study pre-supposes the existence of free mono-energetic electrons at the cathode, whether due to thermionic, field or photo emission. As in case of the classical Child-Langmuir law, the quantum framework merely seeks to predict the maximum steady current that can be transported on application of a field. Importantly, the classical or quantum Child-Langmuir law does not in itself address the question of emission. Together with emission laws however, the limiting Child-Langmuir current plays a role in understanding current-voltage characteristics.

Quantum effects become important at low applied voltages or small gap size and give rise to voltage scaling other than the classical three-halves law $\left(V_{g}^{3 / 2}\right)$, a subject of considerable interest due to the current focus on nanostructured materials. In the deep quantum limit, the small phase volume permits few electrons so that the Hartree potential is inconsequential and the exchange correlation potential dominates. We shall focus on this regime in this communication and try to understand the limiting mechanism and explore the scaling behaviour with respect to the applied potential.

The quantum phenomenon of reflection, tunneling and the fermionic nature of electrons are best incorporated within the Kohn-Sham density functional theory [6] with an effective potential, $V_{\text {eff }}$, that includes a parametrized 
form of the exchange-correlation potential (such as due to Perdew and Zunger [7]) obtained within the local density approximation (LDA). Since, the emission mechanism is not considered here, limitations of LDA, such as the fact that it does not correctly reproduce the image potential, is not of much consequence.

The basic inputs for implementing Kohn-Sham theory to study transport across nanogaps are twofold. The first concerns the boundary conditions for the Hartree potential at the gap boundaries. Since the applied voltage difference is proportional to the difference in chemical potential between the injection and collector planes, the exchangecorrelation potential at the two ends play a significant role in determining the boundary conditions in the deep quantum regime and its neglect can lead to erroneous results 8. The second input lies in the determination of the boundary conditions for the wavefunction and is related to assumptions for the potential beyond the gap for purposes of matching the wavefunction. The simplest of these is to assume that the effective potential assumes a constant value (abruptly) on either side of the gap. This approach is however known to give misleading results when the exchange-correlation potential is neglected altogether 9 . First, an abrupt change in potential for the Schrödinger equation can result in considerable quantum reflection at low applied voltages or small gap size. Further, the coupling of the Schrödinger and Poisson equations makes the boundary conditions for the wavefunction and Hartree potential inconsistent 9]. The problem can be addressed by simply assuming that the transported charged particles are not reflected back into the gap and that the effective potential varies smoothly across the interface. An approximate transmitted wavefunction is then readily available within the semiclassical approximation, which can then be matched with the wavefunction in the nanogap at the collection plane.

Formalism. - We shall first familiarize ourselves with the equations to be solved self-consistently and subsequently arrive at the boundary conditions that they need to satisfy. The effective potential energy, $V_{e f f}$, in the Schrödinger equation

$$
-d^{2} \psi / d x^{2}+V_{e f f} \psi=E \psi
$$

takes the form $V_{e f f}=-e V+V_{x c} \times E_{H}$, where $E_{H}=$ $e^{2} /\left(4 \pi \epsilon_{0} a_{0}\right)$ is the Hartree energy and $a_{0}$ the Bohr radius. The exchange correlation potential within LDA takes the form $V_{x c}=\epsilon_{x c}-\left(r_{s} / 3\right) d \epsilon_{x c} / d r_{s}$, where $r_{s}=[3 /(4 \pi n)]^{1 / 3}$ is the Wigner-Seitz radius, $n$ the electron number density and $e$ the magnitude of the electronic charge. In the above, $V$ is the Hartree potential satisfying the Poisson equation

$$
d^{2} V / d x^{2}=e n(x) / \epsilon_{0}=e|\psi(x)|^{2} / \epsilon_{0}
$$

with boundary conditions that we shall shortly specify. The exchange correlation energy density, $\epsilon_{x c}$ is expressed as $\epsilon_{x c}=\epsilon_{x}+\epsilon_{c}$ where $\epsilon_{x}=-(3 / 2 \pi)^{2 / 3}\left(3 / 4 r_{s}\right)$ is the exchange contribution for a uniform electron gas while $\epsilon_{c}$, the correlation contribution, is represented by a parametrized form of the random phase approximation result. Unless otherwise specified, we shall restrict ourselves to the Perdew-Zunger 7] parametrization.

We first note that the applied voltage difference, $V_{g}=$ $-\left(\mu_{C}-\mu_{E}\right) / e$ where $\mu_{C}$ and $\mu_{E}$ refer respectively to the chemical potential at the collector and injection planes. For convenience, we consider the reference as $\mu_{E}=$ $-e V(0)+V_{x c}(0) \times E_{H}=0$ so that $E=0$ refers to injection from the Fermi level. Thus $V(0)=V_{x c}(0) \times E_{H} / e$. It follows that the chemical potential at the collector is $-e V(D)+V_{x c}(D) \times E_{H}=-e V_{g}$. Thus $V(D)=$ $V_{g}+V_{x c}(D) \times E_{H} / e$. In writing the above, we have implicitly assumed continuity of the chemical potential at the interfaces under steady-state conditions.

The coupled Schrödinger-Poisson system with the above boundary conditions for $V$ are to be solved selfconsistently under the assumption that a current density $J$ is transported across the collection plane. This accounts for one of the two boundary conditions required to solve the Schrödinger equation. The other is derived under an approximation.

The simplest of these is the assumption that the effective potential is constant beyond the collection plane. As discussed in the introduction however, this can lead to erroneous results, mainly because a self-consistent solution requires $V_{\text {eff }}^{\prime}(D)=0$. If the exchange-correlation contribution is small, this implies $V^{\prime}(D)=0$, a condition that is found to be violated except in regimes where a classical description provides an adequate solution.

At the next level, assuming that there is no reflection back into the nanogap and that the effective potential varies sufficiently smoothly at the interface, the transmitted solution can be expressed as a first order WKB wavefunction

$$
\psi_{\text {trans }} \simeq \frac{C}{\sqrt{p(x)}} e^{\frac{i}{\hbar} \int^{x} p\left(x^{\prime}\right) d x^{\prime}}
$$

where $p(x)=\sqrt{2 m\left(E-V_{e f f}(x)\right)}$ is the classical momentum. The constant $C$ can be fixed in terms of the known quantities $J, E$ and $V_{\text {eff }}(D)$. The approximate transmitted wavefunction, $\psi_{\text {trans }}$ can then be used to match the gap wavefunction $\psi$ and its derivative at the boundary.

The coupled system of equations can be solved by first expressing the complex wavefunction

$$
\psi=\sqrt{n_{0}} q(x) e^{i \theta(x)}
$$

in terms of a real amplitude $q(x)$, phase $\theta(x)$ and the characteristic density $n_{0}=2 \epsilon_{0} V_{g} / 3 e D^{2}$. Using dimensionless normalized variables $\bar{x}=x / D, \phi=V / V_{g}, \lambda=$ $D / \lambda_{0}$ where the electron de Broglie wavelength $\lambda_{0}=$ $\sqrt{\hbar^{2} / 2 m e V_{g}}, \epsilon=E / e V_{g}, \bar{J}=J / J_{C L}$ the Schrödinger and Poisson equations can be expressed respectively as [5] 


$$
\begin{gathered}
\frac{d^{2} q}{d \bar{x}^{2}}+\lambda^{2}\left[\epsilon+\phi-\phi_{x c}-\frac{4}{9} \frac{\bar{J}^{2}}{q^{4}}\right] q=0 \quad \text { and } \\
\frac{d^{2} \phi}{d \bar{x}^{2}}=\frac{2}{3} q^{2}
\end{gathered}
$$

where $\phi_{x c}=V_{x c} / \phi_{g}, \phi_{g}=e V_{g} / E_{H}, \quad V_{x c}=\epsilon_{x c}-$ $\left(r_{s} / 3\right) d \epsilon_{x c} / d r_{s}$

$$
\begin{aligned}
\epsilon_{x} & =-\frac{3}{4}\left(\frac{3}{2 \pi}\right)^{2 / 3} \frac{1}{r_{s}} \\
\epsilon_{c} & =\frac{\gamma}{1+\beta_{1} \sqrt{r_{s}}+\beta_{2} r_{s}}
\end{aligned}
$$

with $r_{s}=\left[3 \lambda / 2 \phi_{g} q(\bar{x})\right]^{2 / 3}, \gamma=-0.1423, \beta_{1}=1.0529$ and $\beta_{2}=0.3334$. The parametrized form for the correlation energy density in Eq. 8 is due to Perdew and Zunger [7] with the parameters obtained by fitting to the random phase approximation results.

With the above scaling, the boundary conditions for the scaled Hartree potential (Eq. 6) are

$$
\begin{aligned}
& \phi(0)=\phi_{x c}(0) \\
& \phi(1)=1+\phi_{x c}(1) .
\end{aligned}
$$

The boundary conditions for $q(\bar{x})$ can be derived under the assumption that the transmitted wavefunction is given by Eq. 3 and carries a current density $J$. Using Eq. 3 and $J=e i \hbar\left(\psi^{*} \psi^{\prime}-\psi \psi^{*^{\prime}}\right) / 2 m$, it follows that $|C|^{2}=J m / e$. Without any loss of generality, we shall assume that $C$ is real. Thus $C=\sqrt{J m / e}$. On equating $\psi$ (Eq. 4) and $\psi_{\text {trans }}(\mathrm{Eq} .3 \mathrm{3})$ at $x=D(\bar{x}=1)$, we have

$$
\begin{aligned}
q(1) & =\frac{\sqrt{\frac{2 \bar{J}}{3}}}{\left[\phi(1)+\epsilon-\phi_{x c}(1)\right]^{1 / 4}} \\
& =\frac{\sqrt{\frac{2 \bar{J}}{3}}}{[1+\epsilon]^{1 / 4}}
\end{aligned}
$$

where Eq. 12 follows on using the boundary condition $\phi(1)=1+\phi_{x c}(1)$. In arriving at Eq. 12, we have equated $\theta(1)=(1 / \hbar) \int_{x_{r}}^{x} p\left(x^{\prime}\right) d x^{\prime}$, in effect fixing the value of the reference point $x_{r}$.

The boundary condition for $d q / d \bar{x}$ can be similarly derived by equating the derivatives of $\psi$ and $\psi_{\text {trans }}$. However, since $\phi_{x c}$ depends on $q(\bar{x})$, the value of $q^{\prime}(1)$ must be computed from the equation

$$
q^{\prime}(1)=-\frac{1}{4} \sqrt{\frac{2 \bar{J}}{3}} \frac{\left[\phi^{\prime}(1)-V_{x c}^{\prime}(1) / \phi_{g}\right]}{\left[\phi(1)+\epsilon-\phi_{x c}(1)\right]^{5 / 4}}
$$

where the primes indicate differentiation with respect to $\bar{x}$. Since

$$
\left.\frac{d V_{x c}}{d \bar{x}}\right|_{\bar{x}=1}=\left.\frac{d V_{x c}}{d r_{s}}\right|_{r_{s}(1)} \times\left.\frac{d r_{s}}{d \bar{x}}\right|_{\bar{x}=1}
$$

and

$$
\left.\frac{d r_{s}}{d \bar{x}}\right|_{\bar{x}=1}=-\left(\frac{3 \lambda}{2 \phi_{g}}\right)^{2 / 3} \frac{2}{3} \frac{1}{q^{5 / 3}(1)} q^{\prime}(1),
$$

it follows that

$$
q^{\prime}(1)=-\frac{\frac{1}{4} \sqrt{\frac{2 \bar{J}}{3}} \phi^{\prime}(1)}{[1+\epsilon]^{5 / 4}} \frac{1}{\zeta}
$$

where

$$
\zeta=1+\frac{\left.\frac{1}{6} \sqrt{\frac{2 \bar{J}}{3}}(3 \lambda / 2)^{2 / 3}\left(d V_{x c} / d r_{s}\right)\right|_{r_{s}(1)}}{[1+\epsilon]^{5 / 4}}
$$

As $V_{x c} \rightarrow 0, \zeta \rightarrow 1$ so that $q^{\prime}(1)$ takes the value predicted on ignoring exchange-correlation effects altogether [9].

Voltage Scaling: Dimensional Analysis. - As the Schrödinger equation dictates the deep quantum regime, the scaling with applied voltage should differ from the Poisson equation induced $V_{g}^{3 / 2}$ scaling. The existence of a power law behaviour in the quantum regime cannot be assumed outright. However, numerical results clearly show a power law behaviour in the deep quantum regime (see Fig. 1).

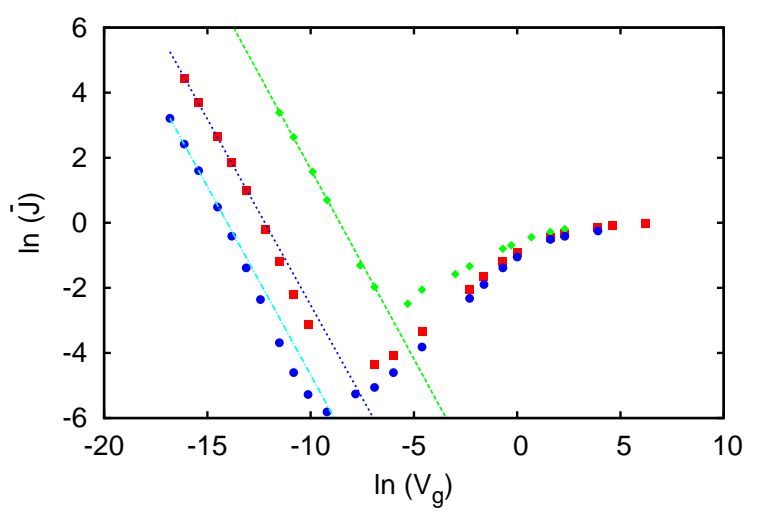

Fig. 1: The maximum scaled current density $\bar{J}$ vs $V_{g}$ for three different nanogaps (i) $D=10 \mathrm{~nm}$ - top set of points marked as diamonds (ii) $D=30 \mathrm{~nm}$ - solid squares in the middle (iii) $D=50 \mathrm{~nm}$ - the bottom set marked by solid circles. The best fitting straight line in the deep quantum regime is also shown. In all three cases, the slope is in the range -1.16 to -1.14 . Note that the voltage at which the deep quantum regime sets in is smaller for larger gaps.

Expressing the scaling in terms of dimensionless quantities as $\bar{J} \sim\left(V_{g} / V_{s}\right)^{\beta}$ where $\bar{J}=J / J_{C L}$ and the voltage scale $V_{s}=\hbar^{2} /\left(2 m e D^{2}\right)$, it follows that

$$
J \sim \frac{\epsilon_{0} e^{\beta+1 / 2} \hbar^{-2 \beta}}{m^{1 / 2-\beta}} \frac{V_{g}^{\beta+3 / 2}}{D^{2-2 \beta}} .
$$


Writing $\beta+3 / 2=\alpha$, it follows that

$$
J \sim \epsilon_{0} \frac{\hbar^{3-2 \alpha} e^{\alpha-1}}{m^{2-\alpha}} \frac{V_{g}^{\alpha}}{D^{5-2 \alpha}}
$$

giving the scaling $J \sim V_{g}^{\alpha} / D^{5-2 \alpha}$ as shown in [9].

The scaling law above is based on numerical observations. It can however be derived from purely dimensional analysis. The relevant equations are the Schrodinger (Eq11) and Poisson equations (Eq. 2) together with the current equation

$$
J=\frac{e \hbar}{2 i m}\left[\psi^{*} \nabla \psi-\psi \nabla \psi^{*}\right] .
$$

Redefining $\tilde{\psi}=\psi / \sqrt{\epsilon_{0}}$, it follows that the Schrodinger and Poisson equations are free of $\epsilon_{0}$ while the current density takes the form

$$
J=\epsilon_{0} \frac{e \hbar}{2 i m}\left[\tilde{\psi}^{*} \nabla \tilde{\psi}^{*}-\tilde{\psi}^{*} \nabla \tilde{\psi}\right] .
$$

Thus, $\tilde{\psi}$ no longer depends on $\epsilon_{0}$ so that $J \sim \epsilon_{0}$. However $\tilde{\psi}$ continues to depend on $e, \hbar$ and $m$ and hence their dependence on $J$ need not be linear. We are now in a position to implement standard dimensional analysis to ascertain the scaling law.

Since, the critical current can in general depend on the quantities $V_{g}, D, \epsilon_{0}, \hbar, m$ and $e$, we demand

$$
J^{-1} V_{g}^{\alpha} D^{\delta_{1}} \hbar^{\delta_{2}} \epsilon_{0} m^{\delta_{3}} e^{\delta_{4}}=C_{0}
$$

where $C_{0}$ is a dimensionless constant. On expressing all the quantities in terms of mass, length, time and current $(M, L, T, A)$, and equating powers of these to zero, we obtain four equations

$$
\begin{aligned}
\delta_{4}-\alpha & =-1 \\
2 \delta_{2}+2 \alpha+\delta_{1} & =1 \\
\delta_{4}-\delta_{2}-3 \alpha & =-4 \\
\delta_{2}+\delta_{3}+\alpha & =1
\end{aligned}
$$

in terms of five unknown quantities. Expressed in terms of $\alpha$, the exponent of $V_{g}$, we obtain $\delta_{1}=2 \alpha-5, \delta_{2}=3-2 \alpha$, $\delta_{3}=\alpha-2$ and $\delta_{4}=\alpha-1$. Thus, standard dimensional analysis gives us Eq. 19 confirming that any power law behaviour must follow this relation.

Note that the Child-Langmuir law follows automatically on demanding that the exponent of $\hbar$ be zero. Thus $\alpha=3 / 2$ so that $J \sim V_{g}^{3 / 2} / D^{2}[10,11$.

Scaling in the deep quantum regime. - While dimensional analysis leads us to the general scaling law $J \sim \hbar^{3-2 \alpha} V_{g}^{\alpha} / D^{5-2 \alpha}$, the value of $\alpha$ observed in Fig. 1] can be understood by the following analysis that involves additional inputs. We first note from Eq. (12) that

$$
\bar{J} \sim q^{2}(1)
$$

To determine the dependence of $q^{2}(1)$ on $V_{g}$, we shall investigate the quantity

$$
\frac{d}{d V_{g}} q^{2}(1)=\left.\frac{d}{d V} q^{2}(\bar{x})\right|_{\bar{x}=1}
$$

where the equality follows on using the alternate but equivalent boundary conditions for the electrostatic potential:

$$
\begin{aligned}
V(\bar{x}=1) & =V_{g} \\
V(\bar{x}=0) & =\frac{E_{H}}{e}\left(V_{x c}(0)-V_{x c}(1)\right)
\end{aligned}
$$

with the injection energy redefined equivalently as $E=$ $V_{x c}(1) E_{H}$. Note that this does not result in any change in the expression for $q(1)$ or $q^{\prime}(1)$ since $\phi(1)+\epsilon-\phi_{x c}(1)$ remains invariant (equals unity) for both sets of boundary condition and injection energy.

Further, since

$$
\frac{d}{d V} q^{2}(\bar{x}=1)=\left(\frac{d}{d \bar{x}} q^{2} / \frac{d}{d \bar{x}} V\right)_{\left.\right|_{\bar{x}=1}}
$$

we have

$$
\begin{aligned}
\frac{d}{d V_{g}} q^{2}(1) & =2 q(1) q^{\prime}(1) / V^{\prime}(1)=\frac{2 q(1) q^{\prime}(1)}{V_{g} \phi^{\prime}(1)} \\
& \sim \frac{\bar{J}}{V_{g} \zeta}
\end{aligned}
$$

We now note that in the quantum regime $\zeta>>1$ so that $\left.\zeta \sim \lambda^{2 / 3} \sqrt{J}\left(d V_{x c} / d r_{s}\right)\right|_{r_{s}(1)}$. As $\lambda \sim V_{g}^{1 / 2}$, it follows that

$$
\frac{d}{d V_{g}} q^{2}(1) \sim \frac{\sqrt{\bar{J}}}{V_{g}^{4 / 3}\left(\frac{d V_{x c}}{d r_{s}}\right)_{\left.\right|_{r_{s}(1)}}} .
$$

Note that the electron density is small at $\bar{x}=1$ compared to $\bar{x}=0$ and is negligible at low applied voltages. Thus $r_{s}(1)$ is large in quantum regime. It can be verified that for the Perdew-Zunger exchange-correlation potential,

$$
\begin{aligned}
\left.\frac{d V_{x c}}{d r_{s}}\right|_{r_{s}(1)} & \sim \frac{1}{r_{s}^{2}(1)} \sim n^{2 / 3}(1) \sim J^{2 / 3} \\
& =\left(\bar{J} J_{C L}\right)^{2 / 3} \sim \bar{J}^{2 / 3} V_{g}
\end{aligned}
$$

Thus,

$$
\frac{d}{d V_{g}} q^{2}(1) \sim \frac{1}{V_{g}^{7 / 3} \bar{J}^{1 / 6}}
$$

Assuming a power law behaviour $\bar{J} \sim V_{g}^{\alpha^{\prime}}$, the above equation translates as

$$
V_{g}^{\alpha^{\prime}-1+\alpha^{\prime} / 6} \sim V_{g}^{-7 / 3} .
$$

On equating the powers, we have $7 \alpha^{\prime} / 6=-4 / 3$, or 


$$
\alpha^{\prime}=-8 / 7 \text {. }
$$

Thus, $\bar{J} \simeq V_{g}^{-8 / 7}$ or $J \sim V_{g}^{5 / 14}$ so that $\alpha=5 / 14$. The value of $\alpha^{\prime}=-8 / 7 \simeq-1.143$ agrees very well with the numerical results presented in Fig. 1 where the exponent $\alpha^{\prime}$ is found to lie between -1.16 and -1.14 in all three cases.

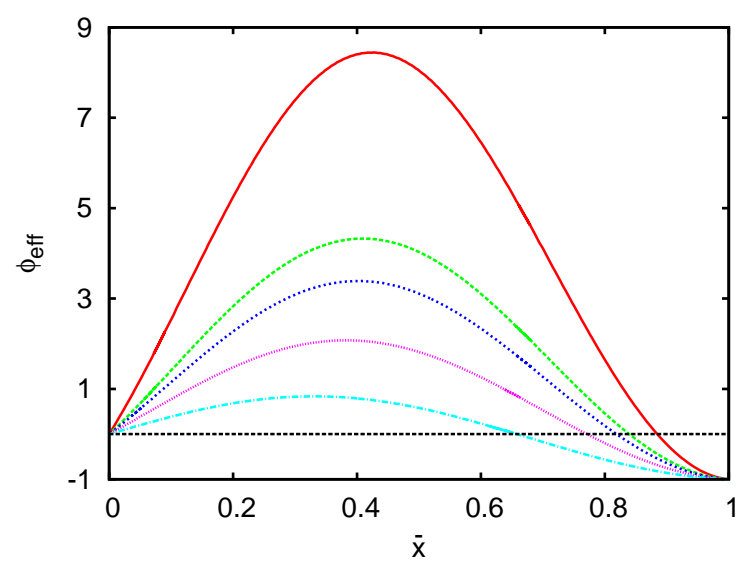

Fig. 2: Typical effective scaled potential curves for $D=30 \mathrm{~nm}$ and $V_{g}=2 \times 10^{-7} \mathrm{~V}$ in order of increasing current density from bottom to top. The horizontal dashed line is $\phi_{\text {eff }}=0$. The values of $\bar{J}$ are 20,30,35,37 and 39.85 (topmost). The critical scaled current density is around 39.9.

The Limiting Mechanism. - In the classical regime, the Schrödinger-Poisson system decouples and the Poisson equation alone dictates the limiting behaviour. In the deep quantum regime of low applied voltages in nanogaps, numerical investigations show that $\phi(\bar{x})$ is linear and follows the applied voltage curve. Thus, the Schrodinger equation alone decides the existence of a physically acceptable solution. We shall briefly consider the limiting mechanism for the model considered in this communication.

In the steady state, an acceptable solution is a smooth, spatially non-oscillatory behaviour of the effective potential. At very low applied voltages in a nanogap, there are too few electrons to alter the electrostatic potential curve from the linear applied potential significantly. Superimposed on this however is the repulsive exchangecorrelation potential which decides the effective potential in the Schrodinger equation. Typically, $\phi_{x c}$ is small at large $r_{s}$ and falls below the electrostatic potential close to the right boundary. Thus the attractive potential dominates near the anode. Near the cathode however, $r_{s}$ is small and $\phi_{x c}$ dominates over $\phi$. Typically, an acceptable limiting effective potential in the deep quantum regime has a barrier towards the cathode, passes through zero and becomes attractive closer to the anode. For $D=30 \mathrm{~nm}$ and $V_{g}=2 \times 10^{-7} \mathrm{~V}$, the change in scaled effective potential with current density is shown in Fig. 2. The topmost potential curve is close to the critical current density. Note that such a solution ceases to exist beyond criticality.
Two observations can be made immediately from the change in potential curves as criticality is approached. First, the barrier height and width increase as the current density is increased. Second, in the region where the electron is free, from the point where it tunnels through the barrier to the collector plate at $\bar{x}=1$, the change in slope increases as criticality is approached (see Fig. 2).

The increase in barrier height and width however cannot be the limiting mechanism for the model considered since even a tiny transmission coefficient cannot violate any of the boundary conditions, in particular that of an outgoing transmitted wave carrying a current density $J$ to the collector. It of course requires a large number of electrons to be present at $\bar{x}=0$, due, for example, to photo-emission.

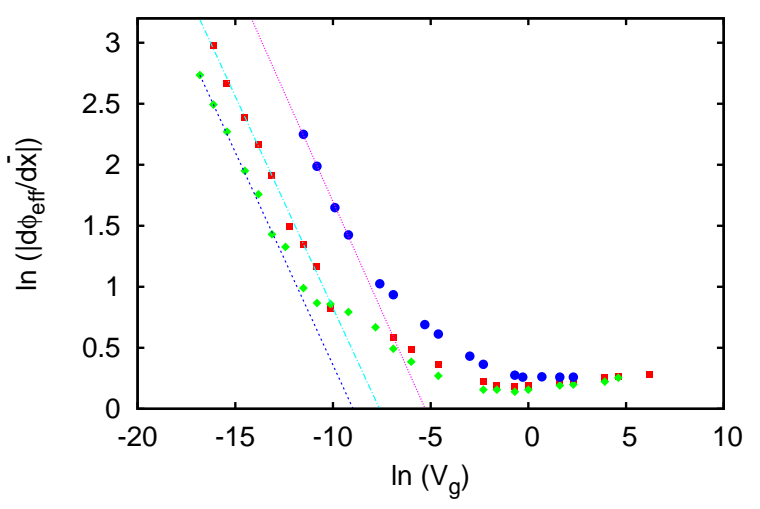

Fig. 3: The maximum slope of the effective potential in the free region vs $V_{g}$ for three different nanogaps (i) $D=10 \mathrm{~nm}$ - top set of points marked as circles (ii) $D=30 \mathrm{~nm}$ - solid squares in the middle (iii) $D=50 \mathrm{~nm}$ - the bottom set marked by diamonds. The best fitting straight line in the deep quantum regime is also shown. In all three cases, the slope is in the range 0.33 0.34. The maximum slope is measured in the region where the electron is free. In the deep quantum regime, the maximum slope occurs at the point where $\phi_{\text {eff }}$ becomes zero while in the classical regime, the maximum slope shifts to $\bar{x}=1$.

A sharp change in slope of the effective potential in the downhill region is however known to cause quantum reflection 13] 14 just as a sudden change in refractive index leads to optical reflection. Such a phenomenon does occur in the approach to criticality as seen in Fig. 2, In order to test the hypothesis, we study the dependence of maximum slope on the applied voltage for different nanogaps in the free region. Fig. 3 shows a universal behaviour in the quantum regime of low applied voltages. The dependence of maximum slope on the applied voltage is clearly identical in all three cases with $\left|d \phi_{e f f} / d \bar{x}\right| \sim V_{g}^{\delta}$ with $\delta \simeq-0.33$ [15. Since the slope $d \phi_{\text {eff }} / d \bar{x} \rightarrow 0$ as $\bar{x} \rightarrow 1$, the onset of criticality appears to be linked to the change in slope seen by the electron as the electron emerges from the barrier. Note that a crucial input in deriving the boundary conditions is the assumption that an outgoing wave carries a 
current density $J$. This breaks down in the presence of quantum reflection from a downhill potential and is possibly the limiting mechanism [16].

Summary and Conclusions. - We have considered the problem of maximum transmitted current on application of a bias, assuming that a sea of electrons exist at the end having lower potential, for example due to photoemission. The quantum model considered is 1-dimensional and includes exchange-correlation effects. The applied bias is carefully fixed in terms of the difference in chemical potential at the two ends and the boundary conditions for the wavefunction amplitude are derived using a WKB expansion at the collector plane to account for a consistent solution of the Poisson-Schrödinger system.

It was shown using standard dimensional analysis that the scaling law applicable across all regimes is $J_{Q C L} \sim$ $\hbar^{3-2 \alpha} V_{g}^{\alpha} / D^{5-2 \alpha}$. The classical Child-Langmuir law follows on demanding that the exponent of $\hbar$ be zero. Thus $\alpha=3 / 2$ and $J_{Q C L} \sim V_{g}^{3 / 2} / D^{2}$.

It was found numerically that for the Perdew-Zunger exchange-correlation potential: (a) the maximum transmitted current falls below the classical prediction till the quantum regime is reached (b) the applied voltage signalling the onset of the quantum regime decreases as the gap size is increased (c) in the deep quantum regime, the maximum transmitted current density is higher than the classical prediction and the region is also marked by a universal behaviour in the scaling of the current density with applied voltage. The voltage scaling was shown analytically to follow $J \sim V_{g}^{\alpha}$ with exponent $\alpha=5 / 14$. The exponent was found to be close to the numerically observed values.

We conclude this communication with a few remarks: (i) the results presented here are directly applicable experimentally only when electrons are made available at the emitter end by an alternate mechanism such as photoemission. In case of field emission, these results can only provide an upper bound to the transmitted current density (ii) the deep quantum regime depends sensitively on the exchange-correlation potential and predictions of higher transmitted current and universal behaviour may vary with a different parametrizations of $V_{x c}$. It may be hoped that careful experimental observations will help decide the appropriate form of of correlation energy applicable for electron transport in nanogaps.

\section{REFERENCES}

[1] C. D. Child, Phys. Rev. Ser. 1 32, 492 (1911); I. Langmuir, Phys. Rev. 2, 450 (1913).

[2] The Child-Langmuir Law for zero injection energy can be generalized in several ways for non-zero injection energy relative to the cathode potential energy [3]. For zeroinjection energy, all coincide.

[3] R. R. Puri, D. Biswas and R. Kumar, Phys. Plasmas 11, $1178(2004)$
[4] Y. Y. Lau, D. Chernin, D. G. Colombant and P.-T. Ho, Phys. Rev. Lett. 66, 1446 (1991).

[5] L. K. Ang, T. J. T. Kwan, and Y. Y. Lau, Phys. Rev. Lett. 91, 208303 (2003).

[6] W. Kohn and L. J. Sham, Phys. Rev. 140 A1133 (1965).

[7] J. P. Perdew and A. Zunger, Phys. Rev. B 23, 5048 (1981)

[8] D. Biswas, Phys. Rev. Lett. 109, 219801 (2012).

[9] D. Biswas and R. Kumar, Eur. Phys. J. B 85189 (2012).

[10] The purported dimensional argument presented in 11 and reiterated in 12 predicts a single value for the exponent $\alpha$ within the Schrödinger-Poisson system and hence does not recover the classical value of $\alpha$ from the quantum formalism. Numerical results however indicate a smooth transition to the classical result.

[11] L. K. Ang, Y. Y. Lau and T. J. T. Kwan, IEEE Trans. Plasma Sci. 32, 410 (2004).

[12] L. .K. Ang, Phys. Rev. Lett. 109, 219802 (2012).

[13] P. L. Garrido, S. Goldstein, J. Lukkarinen and R. Tumulka, Am. J. Phys. 79, 1218 (2011).

[14] B. Segev, R. Côte and M. G. Raizen, Phys Rev A56, R3350 (1997).

[15] Note that classically at criticality, $\phi(\bar{x})=\bar{x}^{4 / 3}$. Thus the points converge at $d \phi_{e f f} / d \bar{x}=4 / 3$ at higher voltages where the classical description is applicable and exchange correlation effects can be neglected.

[16] While a criterion for the onset of quantum reflection in the downhill region has been offered before (see e.g. 14]) and is identical to the criterion for the breakdown of WKB approximation, it is not our intention here to test this or offer a new criterion. 